\title{
OBSERVATIONS ON MICROTHERMAL FLUCTUATIONS
}

\author{
G. R. LYNDS.
}

The concern has been expressed many times by Dr. Bowen and others that a significant portion of the seeing deterioration may occur in levels of the atmosphere very near the ground, within a few tenths of meters of the ground. When I refer to the quality of seeing I am refering to the image size one observes in a telescope of very large aperture and I will assume that this is equivalent to image motion as observed in telescopes of very small aperture. I will not attempt a further justification for this concern; however this is the basis for the studies we are just beginning at Kitt Peak, where we will attempt to quantitatively show whether or not there is need for concern about the very low levels of the atmosphere. So we begin with the thesis that much of the poor seeing observed at a site, the enlargement of photographic or visual images as observed through a large telescope, is due to refractive inhomogeneities in the lower levels of the atmosphere, within less than roo $\mathrm{m}$ above the telescope. We presume that these inhomogeneities are of local origin and that their distribution and motion is determined primarily by site topography, wind direction and velocity. The few experiments we have made thus far at Kitt Peak have been designed to ascertain quantitatively the importance of these factors. Our approach has been to make observations of the large-aperture seeing with simultaneous observations of the thermal structure of the air accessible to us immediately above the telescope.

The observations of the large-aperture seeing are made with the double-beam telescope which has been described by Stock, the same type of instrument currently used in the Chile survey (fig. 4). It has two objectives separated by approximately 60 inches which form two highly magnified images of the same star. The principle, in using the telescope, is to determine the relative motion of these images. At present the telescopes in use are not fitted with drives and the estimates of image motion are made visually. The plan is to equip these telescopes with a sidereal drive and with an electronic device to measure quantitatively and objectively the relative image motion.

The fluctuations in the refractive index of the air above the telescope is being studied by means of observations of its thermal fluctuations. The justification for this is based on the assumption that humidity variations are not a large contributor to index variations and that there are no large aerodynamically induced index refraction fluctuations. We 
also assume some degree of hydrostatic equilibrium and convective stability in the air. Under these conditions you can write for the index of refraction $n$,

$$
d[\ln (n-1)]=d(\ln p)=-d(\ln \mathbf{T})
$$

where $\rho$ is the density and $T$ is the temperature of the air. For $(n-\mathrm{I})=2.83 \times \mathrm{IO}^{-+}$and $\mathrm{T}=\mathrm{IO}^{\circ} \mathrm{C}$ we have

$$
\Delta n=10^{-6} \Delta \mathrm{T} \text {. }
$$

The microthermal measurements are made with resistance thermometers, each consisting of approximately $350 \Omega$ of $25 \mu$ diameter nickel wire. An example of these is shown in figure $2 \mathrm{I}$. The resistance wire is wound in a spiral on a supporting structure of small nylon thread, the spirals being approximately $2 \mathrm{~cm}$ long by $2 \mathrm{~cm}$ in diameter. The resistance wire is operated in a bridge circuit drawing approximately I $\mathrm{mA}$ of current, giving a sensitivity of approximately $2 \mathrm{mV} /{ }^{\circ} \mathrm{C}$. The output from the bridge circuit goes to an amplifier and recorder;



Fig. 21. - A microthermal sensor of the type used in the temperature measurements. The overall height of the unit is about $13 \mathrm{~cm}$. The resistance element is seen as a spiral of $25 \mu$ nickel wire. 
the temperature resolution of the system is of the order of $2.5 \times 1 \mathrm{I}^{-3}{ }^{\circ} \mathrm{C}$. No direct measurements have been made in the laboratory of the bandwidth of the system but several indirect lines of evidence seem to indi-

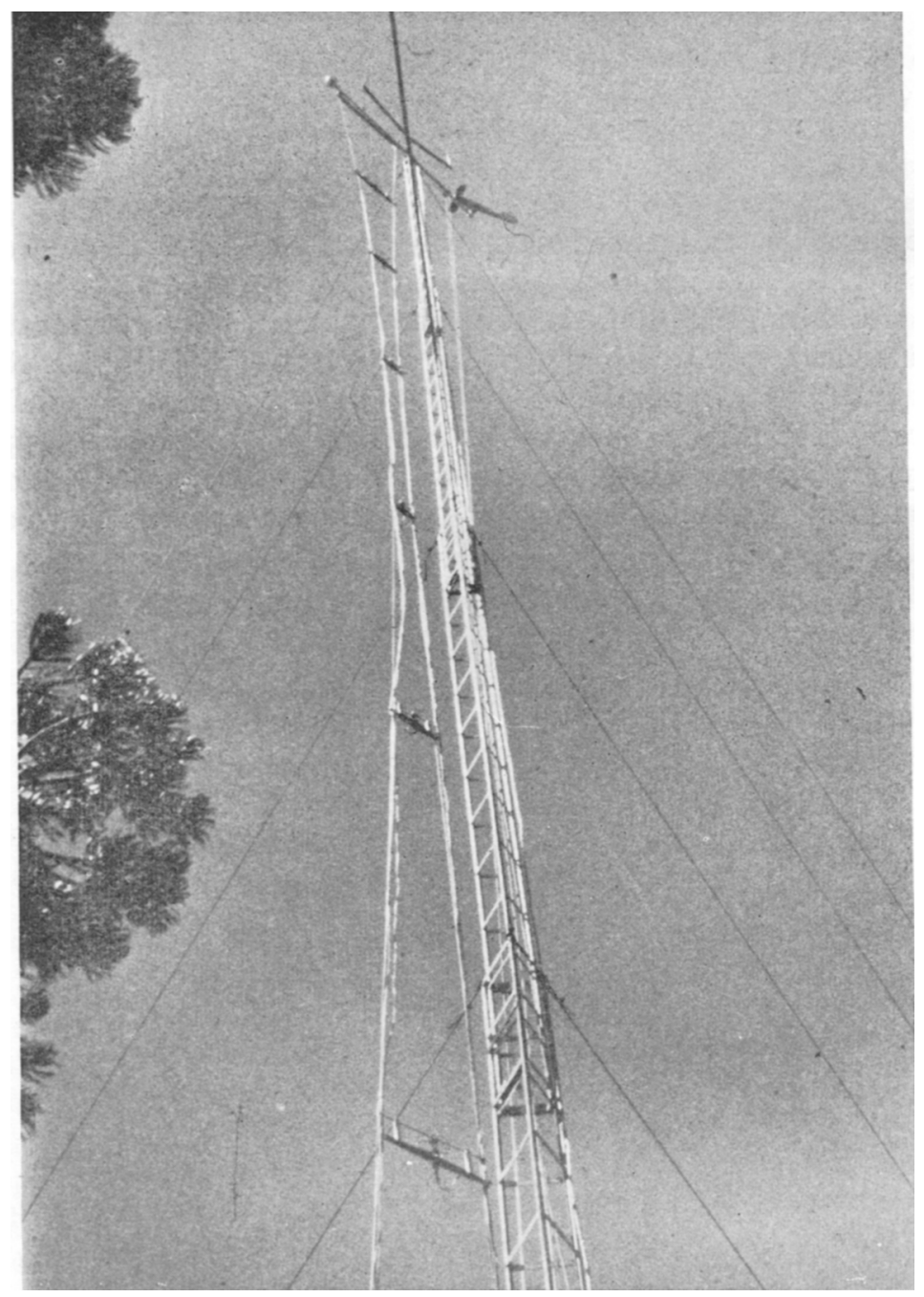

Fig. 22. - An array of seven microthermal sensors extending to a height of $22.5 \mathrm{~m}$.

cate a rise time of ro ms. These thermometers, which we call " microthermal sensors" have been used in a variety of ways. One brief series of observations consisted of observing the temperature fluctuations at approximately $\mathrm{r} .5 \mathrm{~m}$ above the peak of the Kitt Peak 36 -inch dome 
while image quality was estimated visually with the 36 -inch reflector. The observations showed that, on the average, image size was correlated directly with size and frequency of occurence of temperature fluctuations. In addition, individual thermal events were nearly always

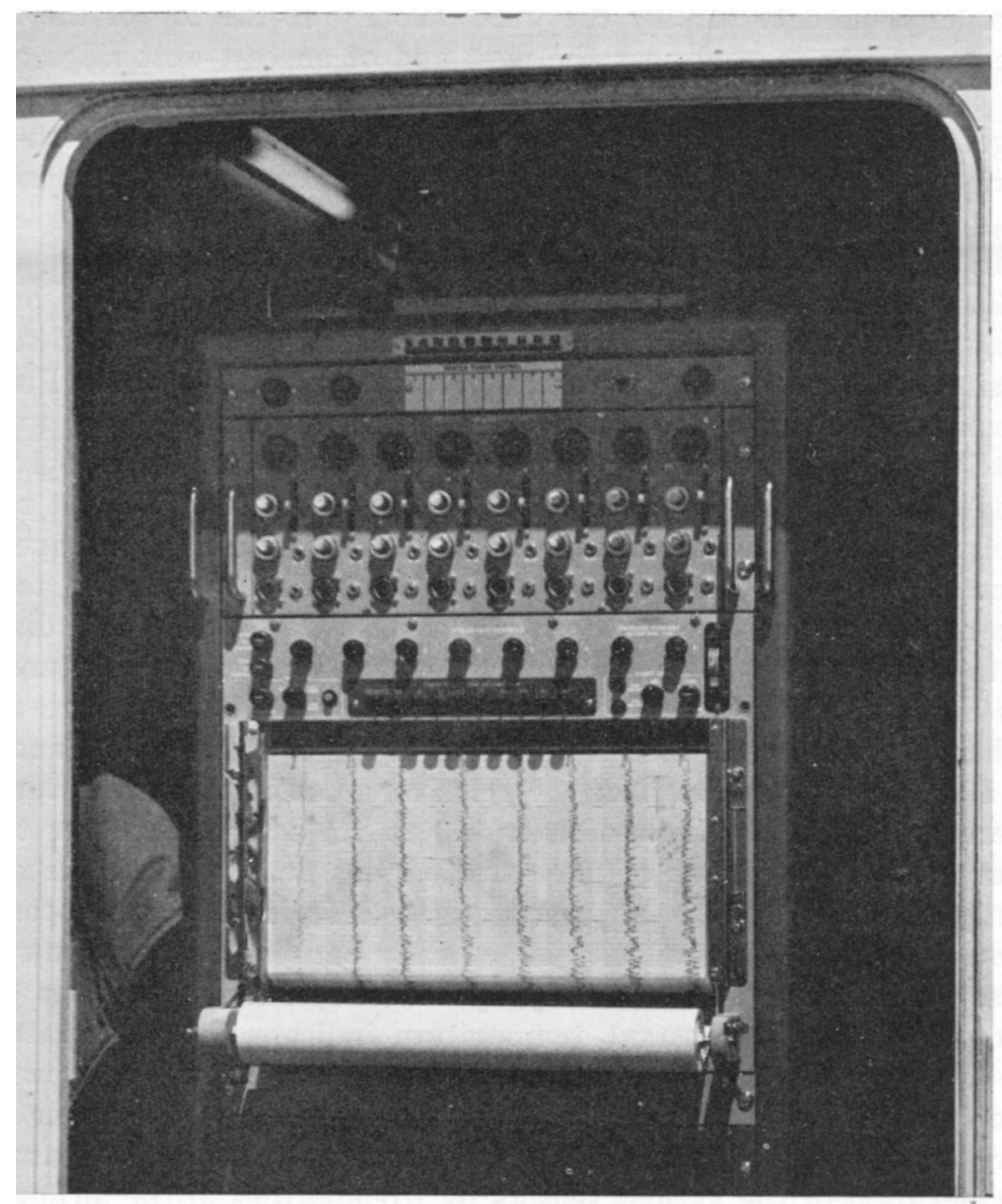

Fig. 23. - An eight-channel recorder which presents temperature fluctuations on seven channel and wind velocity on the remaining channel.

accompanied by poor seeing. (These observations, were facilitated in one instance by the image-orthicon camera which displayed a highly magnified star image.)

The largest part of the program thus far has consisted of an investigation of the vertical spectrum of the temperature variations in the

Symposium U. A. I. no 19. 
air. For these experiments a number of sensors (up to seven) have been arrayed along the side of a vertical tower located on a high point of Kitt Peak. Figure 22 shows the tower and location of the sensors. The fluctuations detected by the sensors are recorded simultaneously on an eight-channel recorder shown in figure 23 . One channel of the recorder is used to display the wind velocity at the top of the tower. The tower was located near the west and of an east-west ridge on the top of Kitt Peak. The location is very near the windward edge of the ridge, the southern edge for prevailing winds from the South-West. During the course of the microthermal measurements, intermittent observations of the seeing were made with a double-beam telescope located about ro m East of the tower.

Since the investigation is still in a preliminary state, I wish only to point out certain characteristics of the microthermal records and suggest possible interpretations. A portion of a record is illustrated in Plate II. The lowest trace shows the wind velocity with full-scale being approximately $10 \mathrm{~m} / \mathrm{s}$. The next seven traces from bottom to top give the temperatures at $4.5,7.5,10.5,16.5,19.5$ and $22.5 \mathrm{~m}$ elevation respectively. The sensitivity corresponds to $5^{\circ} \mathrm{C}$ full-scale. The uppermost trace records I s time marks; time increases from right to left. The time base in the central part of the record is $4 \mathrm{~s}$ per small division. Plate II illustrates the most common type of thermal structure that has been observed thus far. It is found that the temperature at the higher levels is largely free of fluctuations larger than a few hundredth of a degree. These periods of relatively constant temperature are interrupted by fluctuations of from 0.3 to $1.5^{\circ} \mathrm{C}$ towards lower temperatures. The amplitude of these fluctuations does not increase significantly as the ground is approached but their frequency of occurence does. Since the wind velocity is generally between 5 and $10 \mathrm{~m} / \mathrm{s}$ when these conditions prevail, we may hypothesize that the portions of the records showing a constant temperature indicate the temperature of the mass of air flowing over the mountain and that the downward fluctuations in temperature arise from the parcels of cooler air which have been cooled by proximity to the ground and have been carried to the elevation of the various sensors by irregularities in the airflow over the ground. On this picture we would expect, as we approach the ground level, to obtain with our sensors a more and more complete sampling of the cooler air. This is exactly what is found. The record shown in plate III illustrates a similar thermal structure on an expanded time base, ro small divisions per second of time. The parameters for this record are the same as for plate II; the lowest sensor was inoperative for both records.

On some occasions the density of thermal fluctuations remains high, even to the $22.5 \mathrm{~m}$ level; on other occasions, the temperature has been 
found to be constant down to as far as the $7.5 \mathrm{~m}$ level. Normally the scale size of the fluctuation is larger than $20 \mathrm{~cm}$ but on one rare occasion we have observed bursts of high frequency fluctuations indicating scale sizes of less than $10 \mathrm{~cm}$. These are illustrated in plate IV. The time base here is 5o small divisions per second. It is seen that the thermal structure is entirely different from that illustrated in plates II and III. Unfortunately three channels were inoperative at the time this record was taken, but it is still clear that a separate explanation is needed for this type of temperature structure. Some of the high frequency fluctuations observed at about this same time exceeded $5^{\circ} \mathrm{C}$ and were accompanied by very rapid stellar image motion larger than ro seconds of arc.

Regarding possible correlations between seeing and thermal structure, I wish to report only as a qualitative result that the seeing is never good when the thermal fluctuations are large and frequent. Likewise, when the temperature fluctuations are small and infrequent the seeing is generally good, but can occasionnaly be poor.

One can imagine a great variety of experiments which should be performed using the equipment described here. Many of these are planned for the near future. 